\title{
Neuroprotective Effects of Caffeine on a Maternally Separated Parkinsonian Rat Model
}

\author{
Thabisile Mpofana, Willie M. U. Daniels, Musa V. Mabandla \\ Discipline of Human Physiology, School of Laboratory Medicine and Medical Sciences, \\ University of KwaZulu-Natal, Westville Campus, Durban, South Africa \\ Email: $\underline{\text { mabandlam@ukzn.ac.za }}$
}

Received December 4, 2013; revised January 7, 2014; accepted January 16, 2014

Copyright (c) 2014 Thabisile Mpofana et al. This is an open access article distributed under the Creative Commons Attribution License, which permits unrestricted use, distribution, and reproduction in any medium, provided the original work is properly cited. In accordance of the Creative Commons Attribution License all Copyrights @ 2014 are reserved for SCIRP and the owner of the intellectual property Thabisile Mpofana et al. All Copyright (C) 2014 are guarded by law and by SCIRP as a guardian.

\begin{abstract}
Early-life stress has been shown to disrupt the programming of the hypothalamic-pituitary-adrenal (HPA) axis which may have severe consequences in the development of neurological disorders later on in life. Prolonged early-life stressful events produce an exaggerated stress hormone response in the adult offspring. Chronic stress and elevated corticosterone levels have been found to exaggerate functional deficits and accelerate loss of dopamine producing neurons in a rat model of Parkinson's disease. We investigated the neuroprotective effects of caffeine on 6-OHDA lesioned rats that were exposed to maternal separation stress. We examined behaviour of animals before and after the infusion of 6-OHDA using the step and cylinder tests. We also measured dopamine concentration in the striatum, mitochondrial membrane potential in the striatum and the total antioxidant capacity in blood plasma. Maternally separated rats displayed an impaired ability to initiate movement in the step test and a decreased percentage impaired limb use in the cylinder test. In the rats that received caffeine these motor deficits were ameliorated. Maternal separation exaggerated the lesion caused by 6-OHDA injection. However, the neuroprotective effects of caffeine were evident in both the stressed and non-stressed rats as shown by the higher dopamine concentration and total antioxidant capacity on caffeine treated rats. Maternally separated rats had higher mitochondrial membrane permeability when compared to the caffeine treated rats. We therefore conclude that caffeine ameliorated the neurodegeneration associated with 6-OHDA injection in maternally separated animals.
\end{abstract}

\section{KEYWORDS}

Maternal Separation; 6-Hydroxydopamine; Parkinson’s Disease; Behavioural Tests; Caffeine

\section{Introduction}

Stressors early in life have been shown to have enduring effects on the development of the brain [1]. Studies have shown that disruptions in the mother infant relationship increase corticotrophin-releasing factor (CRF) gene expression in the hypothalamus thus affecting the hypothalamic pituitary adrenal (HPA) axis [2,3]. Chronic stress and elevated corticosterone levels have been found to exaggerate functional deficits and accelerate loss of dopamine producing neurons in a rat model for Parkinson's disease [4]. Maternal separation has been used as a model of chronic stress and repeated or long lasting periods of maternal separation during the stress hyporesponsive pe- riod (SHRP) have been shown to alter the stress response [5]. The SHRP is characterized by a low circulating basal glucocorticoid level and therefore maternal separation during this period reduces the sensitivity to glucocorticoid negative feedback [5].

Parkinson's disease is a chronic neurodegenerative disorder of the basal ganglia which is mostly idiopathic in nature [6,7]. This degeneration clinically manifests with motor symptoms which include bradykinesia, muscular rigidity, resting tremor and postural instability [8,9]. Dopamine depletion in the nigral dopamine neurons is the hallmark feature which leads to clinical diagnosis of this disease [10]. Although Parkinson's disease is a typical 
movement disorder, there are many non-motor symptoms associated with the disease which greatly decrease the quality of life [11]. These include cognitive dysfunctions: sleep disorders, psychiatric symptoms such as depression and most commonly gastrointestinal dysfunction [11,12].

Currently, the only therapeutic drugs for Parkinson's disease are symptomatic and such treatments eventually fail. These include the famous L-DOPA treatment which causes drug related dyskinesias after prolonged use [13]. Disease modifying approaches that can slow or stop the progression of neurodegeneration are desperately needed. Many epidemiological studies have linked caffeine consumption with a reduced risk for Parkinson's disease $[14,15]$. The dopamine agonist caffeine provides neuroprotection due to its ability to antagonize Adenosine $\mathrm{A}_{2} \mathrm{~A}$ receptors which reduces adenosine transmission and reverses motor deficits $[16,17]$. In the present study, we investigated the effects of caffeine on the lesion size of a 6-OHDA lesioned rat and whether caffeine provides neuroprotection in a postnatally stressed rat model for Parkinson's disease.

\section{Materials and Methods}

\subsection{Animals}

Forty four male Sprague Dawley rats were used in this study. All rats were obtained from the Biomedical Research Unit and kept under standard lab conditions. All procedures were approved by the Animal Ethics Committee of the University of KwaZulu-Natal (082/13/ Animal). On postnatal day 1, rats were sexed and curled into 6 male pups per litter, incase less than 6 pups, female pups were added to equalize the number of pups per litter. Food and water were freely available for the dams.

\subsection{Maternal Separation}

Rats were randomly divided into two equal groups $(n=22)$ each. One group was normally reared and the second group was maternally separated. The maternal separation protocol started on postnatal day 2 and lasted until postnatal day 14. During this period, the dam was removed from the home cage and placed in a cage with clean bedding. The home cage containing the pups was removed from the housing room and placed in a different room to prevent any form of communication between pups and dams. The separation protocol lasted for 3 hours ( 9 h00 to $12 \mathrm{~h} 00)$. Room temperature was maintained between $31^{\circ} \mathrm{C}$ and $33^{\circ} \mathrm{C}$. After the 3 hours, the cage with the pups was returned to the animal house and the dams were reunited with their pups. The daily light/dark cycle was 6 am to 6 pm. All cages were cleaned once a week. The rats were weaned on postnatal day 21 and kept 4 rats per cage until postnatal day 60 with food and water freely available.

\subsection{Stereotaxic Surgery}

Thirty minutes prior to 6-OHDA infusion, animals were injected with desipramine (15 mg/kg i.p, Sigma, Munich, Germany) a norepinephrine reuptake blocker which serves to prevent 6-OHDA uptake by noradrenergic neurons. Prior to the injection of 6-OHDA, the animals were anaesthetized with sodium pentobarbital $(0.2 \mathrm{ml} / \mathrm{kg}$ i.p, Lakato, South Africa). To aid with respiration during unconsciousness, animals received atropine $(0.2 \mathrm{ml} / \mathrm{kg}$ i.p, Sigma, Munich, Germany) before being placed in the stereotaxic apparatus (David Kopf Instruments, Tujunga, USA). All animals received 6-OHDA $\mathrm{HCl}(5 \mu \mathrm{g} / 4 \mu \mathrm{l}$ dissolved in $0.2 \%$ ascorbic acid; Sigma, St. Louis, MO, U.S.A) infusion unilaterally $(0.5 \mu \mathrm{l} / \mathrm{min})$ using a Hamilton syringe into the right medial forebrain bundle $(4.7 \mathrm{~mm}$ anterior to lambda, $1.6 \mathrm{~mm}$ lateral to midline and $8.4 \mathrm{~mm}$ ventral to dura) (Paxinos and Watson atlas). The infusion needle was left in the medial forebrain bundle for 1 minute prior to the infusion and for a further 5 minutes post infusion in order to allow time for the 6-OHDA to diffuse into the tissue before being slowly retracted from the brain. Post infusion, the burr-hole was closed with silicon wax and the scalp incision was sutured. In order to avoid hypothermia, the rats were placed on a heating pad immediately after surgery and monitored until recovery. The rats were turned from time to time to avoid accumulation of body fluids on one side of the body. Following surgery, the animals were divided into four groups: non-stressed rats (NS), non-stressed rats that received caffeine (NSC), maternally separated rats (MS) and maternally separated rats that received caffeine (MSC) as shown in Table 1 . An hour after surgery, the animals that belonged to the caffeine groups were given their first injection of caffeine (20 mg/kg, i.p, Sigma, Munich, Germany). Once the animals had fully recovered, they were put in cages and taken to the home room. These rats were kept in the home room (two rats per cage) for two weeks (until PND 74) when the post lesion behavioural tests were done.

\subsection{Behavioural Assessment}

On PND 59, the day before stereotaxic lesion all the rats

Table 1. Showing treatment of the four groups, non-stressed rats (NS), non-stressed rats that received caffeine (NSC), maternally separated rats (MS) and maternally separated rats that received caffeine (MSC), $n=11$ per group.

\begin{tabular}{cccc}
\hline Group & MS & 6-OHDA $(5 \mu \mathrm{g} / 4 \mu \mathrm{l})$ & CAFFINE $(20 \mathrm{mg} / \mathrm{kg})$ \\
\hline NS & - & + & - \\
NSC & - & + & + \\
MS & + & + & - \\
MSC & + & + & + \\
\hline
\end{tabular}


were tested for forelimb use symmetry using the cylinder test and the step test. The same tests were repeated two weeks after lesioning (PND 74). After each test the cylinder and the floor were cleaned with $70 \%$ ethanol.

\subsubsection{Forelimb Akinesia (Step Test)}

Rats were assessed for initiation of movement using the step test. The rat was held in position such that the hindquarters and one forelimb (the limb not being tested) were suspended in mid air thus ensuring that the rat supported itself on one forelimb [18]. While held in this position, stepping movements were assessed for each forelimb along a ruler placed on the table and the length of the step taken was recorded. Three steps were taken for each forelimb and the mean was recorded as the length of step taken by each forelimb.

\subsubsection{Forelimb Use Asymmetry Test (Cylinder Test)}

Forelimb use during explorative activity was assessed using the forelimb use asymmetry test. The rats were individually placed in a transparent cylinder and behaviour was recorded by means of a video camera for 5 minutes. We assessed the number of times the animals made independent and simultaneous use of the forelimbs for support, weight shifting movements along the walls and when landing following vertical exploration [18]. The final score was calculated using the formula:

$[($ Right $+1 / 2$ both $)$ divide by ( Right + Left + both $)] \times 100$ with right referring to the limb ipsilateral to the lesioned hemisphere (the non-impaired limb).

\subsection{Sacrifice and Storage of Samples}

Animals were sacrificed on postnatal day 75 using a guillotine. Trunk blood was collected into vacutainer tubes (coated with EDTA to prevent clotting). These tubes were centrifuged at $3500 \mathrm{rpm}$ for 10 minutes at $4^{\circ} \mathrm{C}$ in a refrigerated centrifuge (Z326, Lasec, SA). The plasma was transferred into eppendorfs then stored at $-80^{\circ} \mathrm{C}$ in a biofreezer. Immediately after decapitation the brain was removed and placed in frozen $0.9 \%$ saline slush before the striatum was dissected. The brain tissue was weighed and quickly frozen in liquid nitrogen before being stored in a $-80^{\circ} \mathrm{C}$ bio-freezer where it remained until the day of analysis. All the tissue processing was carefully carried-out on slush.

\subsection{Neurochemimical Analysis}

To validate our behavioural results and establish a neurochemical basis for the differences in behavior, dopamine concentration in the striatum was analysed by ELISA. The total antioxidant capacity in blood was also measured as an indication of cellular damage caused by reactive oxygen species.

\subsubsection{Determination of Striatal Dopamine Levels} Dopamine concentration was measured in the striatum using a dopamine ELISA kit (RE59161, IBL Hamburg, Germany). The striatal tissue was thawed at room temperature and then $500 \mu$ l of EDTA-HCL buffer was added into each microcentrifuge tube containing tissue. The tissue was homogenised with a sonicator (CML-4, Fisher, USA) following which it was centrifuged at $3500 \mathrm{rpm}$ for 10 minutes at $4^{\circ} \mathrm{C}$. The supernatant was pipetted into a new microcentrifuge tube before being stored at $-80^{\circ} \mathrm{C}$ until the day of analysis.

On the day of analysis all samples were thawed at room temperature. The protocol consisted of an extraction and quantification procedure. Both procedures were carried out on the same day.

Extraction: $20 \mu \mathrm{l}$ of each standard, $20 \mu \mathrm{l}$ of each control and $500 \mu \mathrm{l}$ of each sample was pipetted into respective wells in the extraction plate. The standards and controls were then diluted with $500 \mu$ l bidistilled water to correct the differences in volume. All samples, standards and controls were extracted according to the manufacturer's instructions.

Quantification: Extracted standards and controls were diluted with $500 \mu \mathrm{l}$ of release buffer. $100 \mu \mathrm{l}$ of pre-diluted standards and controls and $100 \mu$ l of the extracted samples (without pre-dilution) were pipetted into respective wells containing $75 \mu$ COMT Enzyme solution supplied with the kit. All samples, standards and controls were in duplicate. Optical density was measured with a photometer at $450 \mathrm{~nm}, 60$ minutes after the stop solution was added. For the quantification of dopamine, 6 rats were used per group.

\subsubsection{Total Antioxidant Capacity}

We measured total antioxidant capacity in frozen plasma using the Oxi Select ${ }^{\mathrm{TM}}$ Total Antioxidant Capacity (TAC) Assay Kit (Cellbiolabs San Diego, CA). While thawing the plasma samples, uric acid standards were prepared by serial dilutions from freshly prepared $2 \mathrm{mM}$ uric acid standard. $20 \mu \mathrm{l}$ of the diluted uric acid standards and plasma samples were pipetted into respective wells in a 96 well microplate in duplicate. The reaction buffer was added into each well before mixing by gently shaking. Initial absorbance was obtained by reading the plate at $490 \mathrm{~nm}$. To initiate the reaction, $50 \mu \mathrm{l}$ of $1 \mathrm{x}$ copper ion reagent supplied with the kit was added into each well. After a 5 minutes incubation, $50 \mu$ of $1 \mathrm{x}$ stop solution was added to each well. The plate was read again at 490 $\mathrm{nm}$. To get the net absorbance, the initial reading was subtracted from the final reading and a standard curve using the standards provided was constructed. The concentration of each sample was extrapolated from the standard curve. The concentration was then multiplied by 2189 in order to obtain the copper reducing equivalents (CRE) 
since the assay is a copper based reaction. For total antioxidant capacity 4 rats were used per group.

\subsubsection{Mitochondrial Membrane Potential}

We used freshly dissected brain tissue to measure the mitochondrial membrane potential. For flow cytometric analysis, brain tissue was converted into a cell suspension and a cell count was done by means of haemocytometer to ensure that at least $1 \times 10^{6}$ cells were present in each sample tube for analysis. Cells were stained with JC-1 to determine the mitochondrial membrane potential. $\mathrm{JC}-1$ is a membrane-permeable lipophilic dye that exists as J-aggregates in the mitochondrial matrix and as monomers in the cytoplasm. The tissue was analysed using FacsCalibur flow cytometer (Becton Dickenson, San Jose, CA, USA).

\subsubsection{Statistical Analysis}

The data was analyzed with the software program Graph Pad Prism (version 5) Non-parametric tests (KruskalWallis followed by Mann-Whitney U-test where significance was detected in order to compare all pairs) were used. Data are expressed as mean \pm SEM. Differences were considered significant when $p$-value $<0.05$.

\section{Results}

\subsection{Step Test}

For the step test, we analysed movement initiation in our 4 groups viz (NS, NSC, MS, MSC) $n=11$ in all groups. In the results, unimpaired refers to the limb ipsilateral to the lesion. In both the pre-lesion (Pre) and post lesion (Post) tests all unimpaired limb had a similar step length (results not shown). Maternally separated rats had an impaired ability to initiate movement following 6-OHDA injection *(MS pre vs MS post $p<0.05$; Figure 1). However, this impairment was ameliorated by caffeine injection ${ }^{* *}$ (MS post vs MSC post, $p<0.05$; Figure 1).

\subsection{Cylinder Test}

There was a stress effect as the maternally separated rats that did not receive caffeine (MS) showed a significant decrease in the percentage use of the impaired limb post lesion * (MS pre vs MS post, $p<0.05$; Figure 2). There was also a caffeine effect as maternally separated rats that were treated with caffeine showed an improved ability in the percentage use of the impaired limb when compared to the maternally separated rats that were not treated with caffeine post lesion ${ }^{* *}$ (MS post vs MSC post, $p<0.05$; Figure 2).

\subsection{Dopamine ELISA}

There was a stress effect on dopamine concentration.

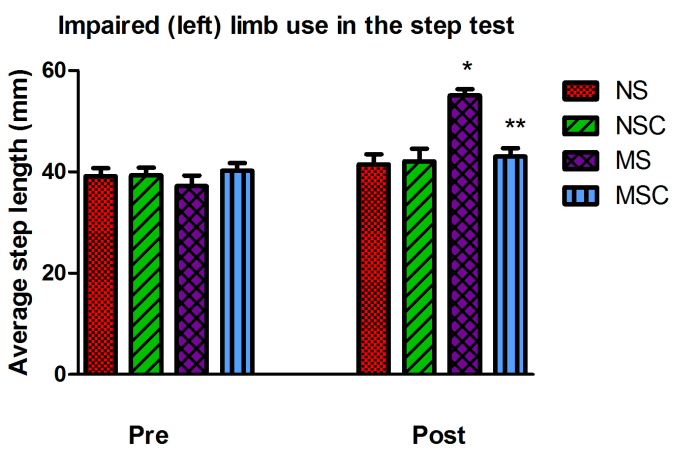

Figure 1. Graph showing the average step length of the left (impaired) forelimb. The result shows both pre and post 6OHDA injection in the non-stressed (NS), non-stressed rats treated with caffeine (NSC), maternally separated (MS) and caffeine treated maternally separated (MSC) rats $(n=11)$. (MS pre vs MS post) $p<0.05,{ }^{* *}$ (MS post vs MSC post) $p<$ 0.05 .

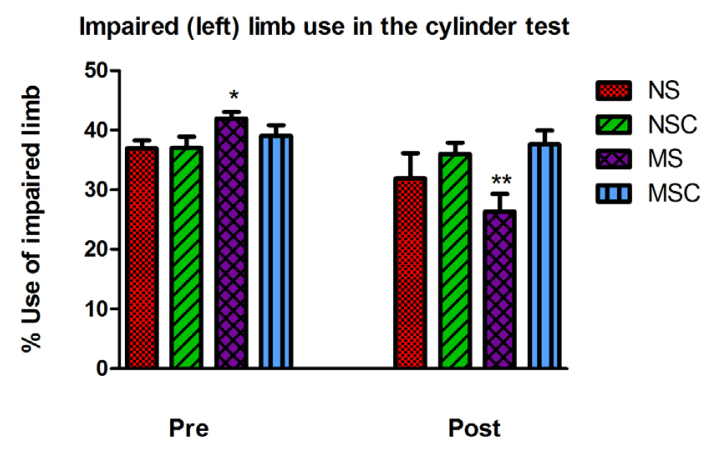

Figure 2. Graph showing percentage use of the impaired limb before (pre) and after (post) 6-OHDA lesion in the nonstressed (NS), non-stressed rats treated with caffeine (NSC), maternally separated (MS) and caffeine treated maternally separated (MSC) rats $(n=11)$. "(MS pre vs MS post) $p<0.05$, (MS post vs MSC post) $p<0.05$.

Maternally separated rats had lower dopamine concentration in the lesioned hemisphere than the non-stressed rats *(NS right vs MS right, $p<0.05$; Figure 3). The lesioned striatum of maternally separated rats had a decreased dopamine concentration when compared to the non-lesioned striatum ${ }^{* *}$ (MS right vs MS left, $p<0.05$; Figure $3)$. There was also a caffeine effect in the lesioned hemisphere of the maternally separated rats as caffeine treated rats had higher dopamine concentration than noncaffeine treated rats ${ }^{* * *}$ (MS right vs MSC right, $p<0.05$; Figure 3).

\subsection{Total Antioxidant Capacity (TAC)}

Our TAC results showed both a stress as well as a caffeine effect on plasma total antioxidant capacity. Maternally separated rats had low TAC concentration than nonstressed rats following 6-OHDA lesion * (NS vs MS, $p<$ 0.05; Figure 4) while this decrease was attenuated by the injection of caffeine ${ }^{* *}$ (MS vs MSC, $p<0.05$; Figure 4). 


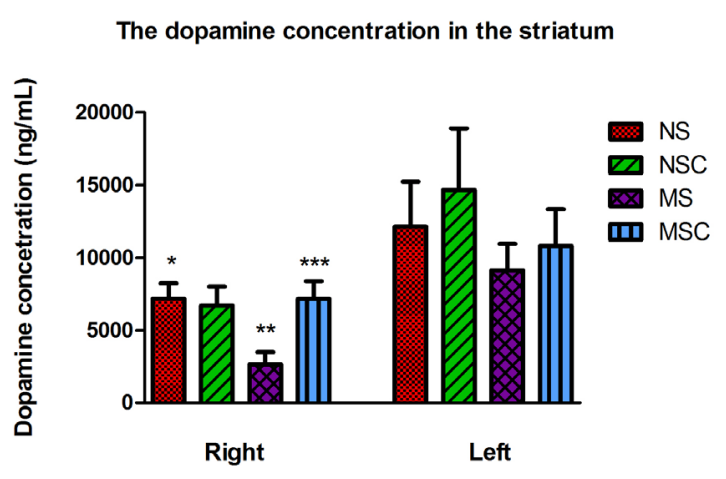

Figure 3. Graph showing dopamine concentration (ng/mL) in the right (lesioned) and left (non-lesioned) striatum of non-stressed (NS), non-stressed rats treated with caffeine (NSC), maternally separated (MS) and caffeine treated maternally separated (MSC) rats $(n=6) . ~ *(N S$ right vs MS right) $p<0.05,{ }^{* *}$ (MS right vs MS left) $p<0.05,^{* * *}$ (MS right vs MSC right) $p<0.05$

The TAC concentration in plasma

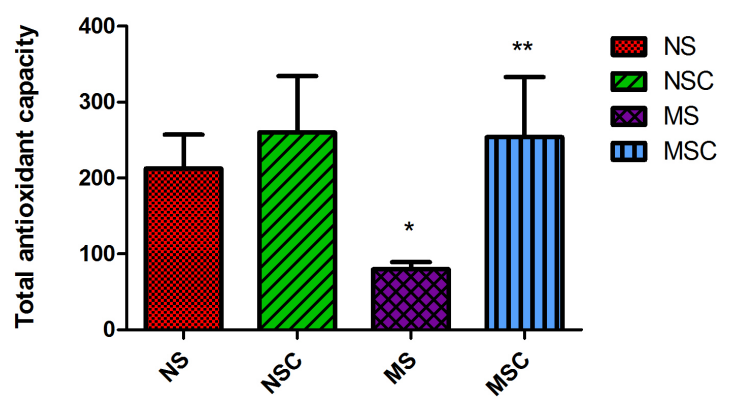

Figure 4. Graph showing total antioxidant capacity in blood of non-stressed (NS), non-stressed rats that received caffeine (NSC), maternally separated (MS) and maternally separated rats that received caffeine (MSC) $(n=4)$. " (NS vs MS) $p<$ $0.05,{ }^{* *}$ (MS vs MSC) $p<0.05$.

\subsection{Mitochondrial Membrane Potential}

There was a stress effect in the mitochondrial membrane potential analysis as the maternally separated rats had a significantly higher percentage cell death in the lesioned striatum when compared to the non-stressed rats * (NS vs MS $p<0.05$; Figure 5). There was a caffeine effect as shown by the decrease in percentage cell death in the caffeine treated maternally separated rats when compared to the non-caffeine rats ${ }^{* *}$ (MS vs MSC, $p<0.05$; Figure $5)$.

\section{Discussion}

6-hydroxydopamine is a well-known neurotoxin that has been widely used to create rodent models of Parkinson's disease $[19,20]$. In our study we injected a preclinical dose of 6-OHDA whose effects were exacerbated by stress (our results show that 6-OHDA lesion alone did not show an effect on the average step length taken by rats using the
Mitochondrial membrane potential on the lesioned striatum

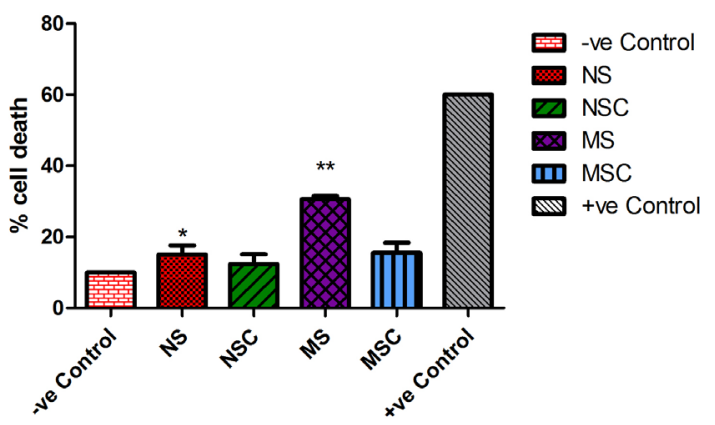

Figure 5. Graph showing Mitochondrial permeability assessed on the lesioned (Right) striatum of non-stressed (NS), non-stressed rats that received caffeine (NSC), maternally separated (MS) and maternally separated rats that received caffeine (MSC) $(n=4)$. Positive control was treated with Chemptothesin (12 $\mu \mathrm{l})$, negative control was an animal infused with saline. "(NS vs MS) $p<0.05,{ }^{* *}$ (MS vs MSC) $p<$ 0.05 .

impaired limb and on the percentage limb use of the impaired limb). Different doses of 6-OHDA injected into the brain produce different stages of this progressive neurodegenerative disease $[19,20]$. When 6-OHDA is injected into the brain it is auto-oxidized and up-taken by catecholamine transporters where it then causes its effects [21]. 6-OHDA inhibits mitochondrial complex 1 resulting to decreased ATP levels and impairment of proton pumping resulting in a decrease in mitochondrial membrane potential which is the first step towards apoptosis [22]. In our study we used a preclinical $(5 \mu \mathrm{l} / 4 \mu \mathrm{g})$ dose of 6-OHDA injected into the right medial forebrain bundle which has been previously shown to produce a mild lesion $[23,24]$. The injection of a mild dose of 6-OHDA did not produce an effect on the behavoiur and neurochemistry of the animals although there were noticeable trends.

However, in the presence of stress, our results showed that the injection of 6-OHDA caused a greater motor impairment. The presence of stress led to a much severe stage of Parkinsonism where motor symptoms were evident on behavoiural testing. An impaired ability to initiate movement and a decreased explorative behavior has been reported following the unilateral injection of 6-OHDA into the medial forebrain bundle [25]. This asymmetry has been associated with a decrease in dopamine concentration. Maternal stress has been shown to increase the risk of developing PD like symptoms in rodent models of PD by affecting the stress response which leads to a greater susceptibility to stress later in life [26,27]. The stress hyporesponsive period (P2-14) in rats is very crucial for the proper development of the nervous system and hence disruptions during this period have been associated with an increased risk of developing psychiatric and neurodegenerative disorders later in life [28]. As shown by our results, maternal separation during the SHRP affected the 
proper development of the HPA axis and stress response which made dopamine neurons more vulnerable to the toxic effects of 6-OHDA [27].

These changes in behaviour where supported by the changes seen in the dopamine concentration in the striatum, mitochondrial membrane potential and total antioxidant capacity in plasma. There was a substantial decrease in dopamine concentration in the lesioned striatum of the maternally separated rats when compared to the non-lesioned hemisphere and to the lesioned hemisphere of the non-stressed rats. Baseline glucocorticoid levels are lower than normal during the SHRP and therefore maternal separation during this period might have exposed the developing neural circuits to high concentrations of glucocorticoids and activation of glucocorticoid receptors (GRs), which subsequently modulates brain and behaviour in later life. Maternally separated rats had higher mitochondrial permeability when compared to the nonstressed rats as an indication of cell death due to apoptosis after the injection of 6-OHDA. In our study we also measured the total antioxidant capacity and our results showed that rats that were maternally separated showed a lower total antioxidant capacity compared to other groups. These results indicate an important role played by the introduction of stressful events like maternal separation in the development and progression of Parkinson's disease suggesting a greater risk of developing severe stages of the disease later in life following early life exposure to stress.

Parkinson's disease is clinically diagnosed when there is more than $80 \%$ dopamine neuron degeneration in the substantianigra [29]. Risk factors associated with Parkinson's disease include age as the major contributor; however, environmental factors have been shown to play a role in the development of Parkinson's disease [26].

Caffeine intervention attenuated the toxic effects of 6OHDA in the maternally separated rats. This caffeine effect was evident in the behavioural tests as well as in dopamine degeneration, mitochondrial membrane potential and total antioxidant capacity of caffeine treated rats. Caffeine treated rats had a higher dopamine concentration in the striatum compared to caffeine naïve rats. This is an important result as caffeine has been shown to attenuate the effects of neurotoxins in rodent models of Parkinson's disease [30]. Studies have shown that caffeine provides neuroprotection due to its ability to antagonize adenosine receptors and thus reduces adenosine transmission and reverse motor deficits [31,32]. This was also shown in the mitochondrial membrane potential where caffeine treated rats had a lower percentage of compromised mitochondria while caffeine treated rats were not different from the negative control.

An inverse association between plasma total antioxidant capacity and neurological damage has been estab- lished [33]. Caffeine treated rats also showed a significantly higher total antioxidant capacity in plasma as compared to the non-treated rats. This may be due to the ability of caffeine to increase the production of antioxidants such as glutathione which has strong free radical scavenging properties as shown by Aoyama et al., [34]. Low antioxidant capacity indicated higher neurotoxin induced cell damage while higher antioxidant capacity was associated with low free radicals. It is important to note that caffeine effect was also present in the maternally separated rats. Our results show caffeine to provide neuroprotection in a stressed model of Parkinson's disease.

\section{Conclusion}

Maternal separation plays a major role in affecting proper development of the neurocircuitry. These disruptions early in life have enduring effects and predispose the rat to be particularly vulnerable to neurodegeneration later in life. Maternal separation exacerbated the effects of 6OHDA as shown by the asymmetry on movement initiation, explorative activity supported by the reduced dopamine concentration and increased mitochondrial permeability. Caffeine and other dopamine agonists promise to be the breakthrough in the treatment of Parkinson's disease. Caffeine ameliorated neurodegeneration associated with 6-OHDA toxicity and its ability to ameliorate these effects in the maternally separated rats shows a possible neuroprotective effect of caffeine even in more severe stages of Parkinson's disease.

\section{Acknowledgements}

The authors would like to thank the National Research Foundation for financial support, as well as the staff of the Biomedical Resource Center of the University of KwaZulu-Natal for technical assistance.

\section{REFERENCES}

[1] Y. Pan, Y. Liu, K. A. Young, Z. Zhang and Z. Wang, "Post-Weaning Social Isolation Alters Anxiety-Related Behavior and Neurochemical Gene Expression in the Brain of Male Prairie Voles,” Neuroscience Letters, Vol. 454, No. 1, 2009, pp. 67-71. http://dx.doi.org/10.1016/j.neulet.2009.02.064

[2] C. E. Grace, S. Kim and J. M. Rogers, "Maternal Influences on Epigenetic Programming of the Developing Hypothalamic-Pituitary-Adrenal Axis,” Birth Defects Research (Part A), Vol. 91, No. 8, 2011, pp. 797-805.

[3] M. Nishi, N. Horii-Hayashi, T. Sasagawa and W. Matsunaga, "Effects of Early Life Stress on Brain Activity: Implications from Maternal Separation Model in Rodents," General and Comparative Endocrinology, Vol. 181, 2013, pp. 306-309.

http://dx.doi.org/10.1016/j.ygcen.2012.09.024 
[4] L. K. Smith, N. M. Jadavji, K. L. Colwell, S. K. Perehudoff and G. A. Metz, "Stress Accelerates Neural Degeneration and Exaggerates Motor Symptoms in a Rat Model of Parkinson's Disease," European Journal of Neuroscience, Vol. 27, No. 8, 2008, pp. 2133-2146. http://dx.doi.org/10.1111/j.1460-9568.2008.06177.x

[5] G. Biagini, E. M. Pich, C. Carani, P. Marrama and L. F. Agnati, "Postnatal Maternal Separation during the Stress Hyporesponsive Period Enhances the Adrenocortical Response to Novelty in Adult Rats by Affecting Feedback Regulation in the CA1 Hippocampal Field,” International Journal of Developmental Neuroscience, Vol. 16, No. 3-4, 1998, pp. 187-197. http://dx.doi.org/10.1016/S0736-5748(98)00019-7

[6] A. Wood-Kaczmar, S. Gandhi and N. W. Wood, "Understanding the Molecular Causes of Parkinson's Disease,” TRENDS in Molecular Medicine, Vol. 12, No. 11, 2006, pp. 521-528.

http://dx.doi.org/10.1016/j.molmed.2006.09.007

[7] F. Blandini, G. Nappi, C. Tassorelli and E. Martignoni, "Functional Changes of the Basal Ganglia Circuitry in Parkinson's Disease,” Progress in Neurobiology, Vol. 62, No. 1, 2000, pp. 63-88. http://dx.doi.org/10.1016/S0301-0082(99)00067-2

[8] B. Goren, Z. Mimbay, N. Bilici, M. Zarifoglu, E. Ogul and E. Korfal, "Investigation of Neuroprotective Effects of Cyclooxygenase Inhibitors in the 6-Hydroxydopamine Induced Rat Parkinson Model,” Brain Research, Vol. 19, No. 3, 2009, pp. 230-236.

[9] A. M. Hemmerle, J. P. Herman and K. B. Seroogy, "Stress, Depression and Parkinson's Disease," Experimental Neurology, Vol. 233, No. 1, 2012, pp. 79-86. http://dx.doi.org/10.1016/j.expneurol.2011.09.035

[10] W. G. Ondo, "Motor Complications in Parkinson's Disease," International Journal of Neuroscience, Vol. 121, No. 2, 2011, pp. 37-44. http://dx.doi.org/10.3109/00207454.2011.620198

[11] T. M. Armentero, A. Pinna, S. Ferré, J. L. Lanciego, C. E. Müller and R. Franco, "Past, Present and Future of A2A Adenosine Receptor Antagonists in the Therapy of Parkinson's Disease," Pharmacology \& Therapeutics, Vol. 132, No. 3, 2011, pp. 280-299. http://dx.doi.org/10.1016/j.pharmthera.2011.07.004

[12] W. Poewe, "Non-Motor Symptoms in Parkinson's Disease,” European Journal of Neurology, Vol. 15, 2008, pp. 14-20.

http://dx.doi.org/10.1111/j.1468-1331.2008.02056.x

[13] Y. Smith, T. T. Wichman, S. A. Factor and M. R. DeLong, "Parkinson's Disease Therapeutics: New Developments and Challenges since the Introduction of Levodopa,” Neuropsychopharmacology REVIEWS, Vol. 37, No. 1, 2012, pp. 213-246. http://dx.doi.org/10.1038/npp.2011.212

[14] K. Xu, Y. Xu, D. Brown-Jermyn, J. Chen, A. Ascherio, D. E. Dluzen and M. A. Schwarzschild, "Estrogen Prevents Neuroprotection by Caffeine in the Mouse 1-Methyl-4Phenyl-1,2,3,6-Tetrahydropyridine Model of Parkinson's Disease," The Journal of Neuroscience, Vol. 26, No. 2, 2006, pp. 535-541.

http://dx.doi.org/10.1523/JNEUROSCI.3008-05.2006
[15] S. E. Seidl and J. A. Potashkin, "The Promise of Neuroprotective Agents in Parkinson's Disease," Neuroprotection in Parkinson's Disease, Vol. 2, No. 68, 2011, pp. 119.

[16] A. Ho, "Two Wrongs Make A Right: Nicotine and Caffeine as Defensive Agents against Parkinson's Disease," Nutrition Bytes, Vol. 8, No. 2, 2002, pp. 1-8. http://escholarship.org/uc/item/7bz2q9rn

[17] R. E. Moo-Puc, J. L. Góngora-Alfaro, F. J. Alvarez-Cervera, J. C. Pineda, G. Arankowsky-Sandoval and $\mathrm{H}$. López, "Caffeine and Muscarinic Antagonists Act in Synergy to Inhibit Haloperidol-Induced Catalepsy,” Neuropharmacology, Vol. 45, No. 4, 2003, pp. 493-503. http://dx.doi.org/10.1016/S0028-3908(03)00202-8

[18] J. L. Tillerson, A. D. Cohen, J. Philhower, G. W. Miller, M. J. Zigmond and T. Schallert, "Forced Limb-Use Effects on the Behavioural and Neurochemical Effects of 6-Hydroxydopamine,” The Journal of Neuroscience, Vol. 21, No. 12, 2001, pp. 4427-4435.

[19] U. Ungerstedt, "Postsynaptic Supersensitivity after 6-Hydroxy-Dopamine Induced Degeneration of the NigroStriatal Dopamine System,” Acta Physiological Scandinavica Supplementum, Vol. 367, 1971, pp. 69-93.

[20] H. Yuan, S. Sarre, G. Ebinger and Y. Michotte, "Histological, Behavioural and Neurochemical Evaluation of Medial Forebrain Bundle and Striatal 6-OHDA Lesions as Rat Models of Parkinson's Disease," Journal of Neuroscience Methods, Vol. 144, No. 1, 2005, pp. 35-45. http://dx.doi.org/10.1016/j.jneumeth.2004.10.004

[21] H. Thoenen and J. P. Tranzer, “Chemical Sympathectomy by Selective Destruction of Adrenergic Nerve Endings with 6-Hydroxydopamine," Naunyn-Schmiedeberg's Archives of Pharmacology, Vol. 261, No. 3, 1968, pp. 271288. http://dx.doi.org/10.1007/BF00536990

[22] Y. Y. Glinka and M. B. Youdim, "Inhibition of Mitochondrial Complexes I and IV by 6-Hydroxydopamine," European Journal of Pharmacology, Vol. 292, No. 3-4, 1995, pp. 329-332.

[23] M. J. Zigmond and E. M. Stricker, "Animal Models of Parkinsonism Using Selective Neurotoxins; Clinical and Basic Implications," International Review of Neurobiology, Vol. 31, No. 1, 1989, pp. 2-60.

[24] E. M. Emborg, "Evaluation of Animal Models of Parkinson's Disease for Neuroprotective Strategies," Journal of Neuroscience Methods, Vol. 139, No. 2, 2004, pp. 121143. http://dx.doi.org/10.1016/j.jneumeth.2004.08.004

[25] T. Schallert and M. Woodlee, "Motor System: Orienting and Placing," In: I. Q. Whishaw and B. Kolb, Eds., The Behaviour of the Laboratory Rat: A Handbook with Tests, Oxford University Press, New York, 2005, pp. 129-140.

[26] F. Curulli, A. Berry and E. Alleva, "Early Disruption of the Mother-Infant Relationship: Effects on Brain Plasticity and Implications for Psychopathology,” Neuroscience and Biobehavioral Reviews, Vol. 27, No. 1, 2003, pp. 7382. http://dx.doi.org/10.1016/S0149-7634(03)00010-1

[27] B. A. Morrow, R. H. Roth, D. E. Redmond Jr., S. Diano and J. D. Elsworth, "Susceptibility to a Parkinsonian Toxin Varies during Primate Development,” Experimental Neurology, Vol. 235, No. 1, 2012, pp. 273-281. 
http://dx.doi.org/10.1016/j.expneurol.2012.02.005

[28] M. R. Gunnar, "Integrating Neuroscience and Psychological Approaches in the Study of Early Experiences," Annals of the New York Academy of Sciences, Vol. 1008, No. 1, 2003, pp. 238-247. http://dx.doi.org/10.1196/annals.1301.024

[29] S. Hunot, F. Boissiere, B. Faucheux, B. Brugg, A. Mouatt-Prigent, Y. Agid and E. C. Hirsch, "Nitric Oxide Synthase and Neuronal Vulnerability in Parkinson's Disease," Neuroscience, Vol. 72, No. 2, 1996, pp. 355-363. http://dx.doi.org/10.1016/0306-4522(95)00578-1

[30] P. K. Sonsalla, L. Y. Wong, S. L. Harris, J. R. Richardson, I. Khobahy, W. Li, B. S. Gadad and D. C. German, "Delayed Caffeine Treatment Prevents Nigral Dopamine Neuron Loss in a Progressive Rat Model of Parkinson's Disease," Experimental Neurology, Vol. 234, No. 2, 2012, pp. 482-487.

http://dx.doi.org/10.1016/j.expneurol.2012.01.022

[31] J. F. Chen, K. Xu, J. P. Petzer, R. Staal, Y. H. Xu, M. Beilstein, P. K. Sonsalla, K. Castagnoli, N. Castagnoli Jr. and M. A. Schwarzschild, "Neuroprotection by Caffeine and A2A Adenosine Receptor Inactivation in a Model of Parkinson's Disease," The Journal of Neuroscience, Vol. 21, No. 10, 2001, p. 143.

[32] M. Morelli, A. R. Carta, A. Kachroo and M. A. Schwarzschild, "Pathophysiological Roles for Purines: Adenosine, Caffeine and Urate,” Progress in Brain Research, Vol. 183, 2010, pp. 183-208. http://dx.doi.org/10.1016/S0079-6123(10)83010-9

[33] J. S. Leinonen, J. Ahonen, K. Lönnrot, M. Jehkonen, P. Dastidar, G. Molnár and H. Alho, "Low Plasma Antioxidant Activity Is Associated with High Lesion Volume and Neurological Impairment in Stroke,” Stroke, Vol. 31, No. 1, 2000, pp. 33-39. http://dx.doi.org/10.1161/01.STR.31.1.33

[34] K. Aoyama, N. Matsumura, M. Watabe, F. Wang, K. Kikuchi-Utsumi and T. Nakaki, "Caffeine and Uric Acid Mediate Glutathione Synthesis for Neuroprotection,” Neuroscience, Vol. 181, 2011, pp. 206-215. http://dx.doi.org/10.1016/j.neuroscience.2011.02.047 